Commun:HEIT! Communication et organisation

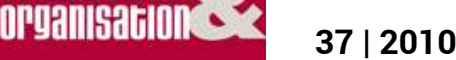

La communication à l'épreuve des mutations économiques

\title{
Consumer Culture Theory et Marketing
}

Une ouverture pour les SIC?

\section{Benoit Cordelier}

\section{CpenEdition}

Journals

Édition électronique

URL : http://journals.openedition.org/communicationorganisation/1323

DOI : 10.4000/communicationorganisation. 1323

ISSN : 1775-3546

Éditeur

Presses universitaires de Bordeaux

Édition imprimée

Date de publication : 1 juin 2010

Pagination : 165-177

ISBN : 978-2-86781-710-6

ISSN : $1168-5549$

Référence électronique

Benoit Cordelier, "Consumer Culture Theory et Marketing ", Communication et organisation [En ligne],

37 | 2010, mis en ligne le 26 avril 2011, consulté le 30 avril 2019. URL : http://journals.openedition.org/ communicationorganisation/1323 ; DOI : 10.4000/communicationorganisation. 1323 


\title{
Consumer Culture Theory et Marketing Une ouverture pour les SIC?
}

\author{
Benoît Cordelier ${ }^{1}$
}

Le marketing et la manière dont y est traitée la communication sont souvent critiqués en Sciences de l'information et de la communication (SIC) ${ }^{2}$ (Floris, 1998; 2001; Dacheux, 2001). Sa finalité instrumentale et l'approche positiviste et déterministe qui y sont généralement adoptées ne correspondent pas aux canons d'une interdiscipline qui s'inscrivent plus volontiers dans une perspective herméneutique, compréhensive et complexe (Courbet, 2001; Bernard \& Joule, 2005). Les recherches sur la publicité, par exemple, convoquent différents domaines en Sciences humaines et sociales ayant déjà participé à la construction des SIC telles que la littérature, la philosophie, la sémiologie et les approches politiques (Courbet, 2001). Pourtant, dans notre discipline, le marketing est plus souvent étudié comme un objet de recherche en soi, voire condamné à travers une posture critique. Cette dernière est d'ailleurs encore forte en SIC. Elle a été impulsée par la sociologie critique de Francfort et l'influence d'Habermas qui nous font percevoir «dans le marketing tout à la fois un instrument de manipulation destiné à fabriquer "une loyauté de masse" (Habermas, 1978) et un outil de légitimation du système capitaliste (Ewen, 1983)» (Dacheux, 2001). Le marketing est donc un outil de manipulation ou relève, au mieux de la communication persuasive. Il pourrait dès lors sembler difficile d'investir les objets de la communication marketing en les partageant avec les Sciences de gestion (SG). Pourtant, depuis un peu plus d'une vingtaine d'années ( $c f$. Arnould \& Thompson, 2005), un courant en recherche du consommateur s'est développé aux Etats-Unis sur des bases épistémologiques et méthodologiques proches de celles des SIC, le Consumer

01. Benoit Cordelier est professeur au Département de communication sociale et publique de l'Université du Québec à Montréal (UQAM). Directeur de l'unité de programmes en communication publique (baccalauréats en relations publiques et en communication marketingl et du Centre d'études en communications marketing, ses recherches portent sur le changement et la structuration des organisations avec les systèmes d'information ainsi que sur la marchandisation du lien social dans les communautés virtuelles. Mail : cordelier.benoił@uqam.ca

02. L'acronyme usuel en France, "SIC », sera adopté tout au long de l'article pour Sciences de l'Information et de la Communication. 
Culture Theory ${ }^{3}$. La ou les CCT ne se présentent pas comme un grand ensemble théorique intégré, mais plutôt comme une étiquette regroupant de nombreuses perspectives théoriques s'intéressant aux cultures dans lesquelles se meuvent les consommateurs, à leur relation au marché, aux phénomènes de consommation (Arnould \& Thompson, 2005). L'appellation est récente et peut donc encore être bousculée (Arnould \& Thompson, 2005, 2007; Moisander, Peñalosa \& Valtonen, 2009). Elle est d'ailleurs réellement controversée, dans le milieu même où elle se développe (Arnould, 2006). Et d'autres étiquettes, comme celle d'Interpretive Consumer Research, sont proposées. Il n'en reste pas moins qu'elle répond à un besoin de mise en visibilité institutionnelle, car si ses chercheurs sont en général rattachés à des départements de marketing ou à des écoles de gestion, leur formation, leurs intérêts prennent source dans les Sciences humaines et sociales et leurs approches ne correspondent pas aux courants dominants des SG.

Les intérêts théoriques des chercheurs en CCT portent autour de la notion de culture que nous savons depuis longtemps polysémique et complexe ( $c f$. Kroeber et Kluckhohn, 1952; Cuche, 2004). La culture est donc approchée dans ce courant comme un ensemble hétérogène de représentations, de valeurs partagées et de modes de vie propres aux membres d'une communauté. Celle des consommateurs devient le reflet d'une construction sociale mobilisant des ressources matérielles et symboliques fournies et relayées par le marché et les médias. Il devient dès lors évident que ce courant peut nous permettre d'apercevoir des pistes de recherche en marketing que peuvent investir les SIC sans s'enfermer dans les approches critiques ou simplement descriptives auxquelles elles sont plus habituées dans ce domaine. Notre objectif dans cet article est par conséquent de présenter un point de vue, forcément partiel, des CCT et de mettre en évidence quelques éléments de convergence avec les SIC. Dans un premier temps, nous présenterons donc les origines institutionnelles des CCT, puis nous en exposerons les grands axes de recherche tels qu'identifiés par Arnould \& Thompson (2005, 2007). Enfin, nous nous pencherons sur quelques similitudes dans le cheminement épistémologique entre SIC et CCT.

\section{Origines institutionnelles}

Ce qui allait devenir les CCT est apparu de la volonté de chercheurs d'échapper aux approches du marketing marquées par la micro-économie, la psychologie cognitive, les recherches expérimentales et les méthodes quantitatives. Allions-nous assister à une rupture avec les sciences de gestion, à un schisme? Cela ne semble pas être le cas. En effet, Arnould \& Thompson (2007) estiment à autour de $4 \%$ les chercheurs sympathisants des CCT dans les départements de marketing des 20 meilleures universités américaines.

03. Ou Consumer Culture Theoretics (Arnould \& Thompson, 2007) ; dorénavant, nous emploierons l'acronyme CCT en tant que pluriel. 
Bien qu'ils se considèrent comme une minorité mal entendue, cela laisse présager plutôt une reconfiguration dans la discipline qu'une séparation. La construction de cette communauté épistémique se veut alors un travail de mise en visibilité (Arnould \& Thompson, 2005), voire un "acte de pouvoir » (Moisander, Peñalosa \& Valtonen, 2009, p. 9).

Dans la francophonie ${ }^{4}$, les chercheurs qu'Özçağlar-Toulouse \& Cova (2008) identifient comme faisant partie des CCT sont aussi pour l'essentiel institutionnellement ancrés dans des départements de marketing ou des écoles de gestion. Ce constat un peu rapide ne doit pas pour autant masquer le fait que ces chercheurs ont su assimiler des apports disciplinaires externes telles l'anthropologie ou la sémiotique. Autre exemple parlant, parmi les figures de proue de la mouvance française nous avons Olivier Badot qui avant d'être professeur en marketing est docteur en anthropologie. Les CCT aussi bien francophones (Özçağlar-Toulouse \& Cova, 2008) qu'anglophones (Arnould $\&$ Thompson, 2007) se perçoivent d'ailleurs comme une interdiscipline.

Les deux courants se construisent en s'inspirant du renouveau ${ }^{5}$ que propose le postmodernisme avec des auteurs comme Lipovetsky, Lyotard, Baudrillard ou encore Maffesoli. Cette influence est controversée au point que des penseurs qui y sont associés peuvent rejeter cette approche (Deleuze, Derrida). Les CCT dans le marketing n'échappent pas à ce débat; Arnould \& Thompson $(2007$, p. 4) la qualifient d'ailleurs le postmodernisme de " trou noir conceptuel». Mais cette influence permet pourtant de s'écarter du marketing positiviste (Brown, 1993; Arnould \& Thompson, 2005, 2007; Özçağlar-Toulouse \& Cova, 2008). La structuration du courant se fait progressivement au fur et à mesure que se développe une communauté épistémique (Moisander, Peñalosa \& Valtonen, 2009).

La reconnaissance de la pluralité théorique semble être alors la condition nécessaire au développement d'un ensemble de chercheurs qui se caractérise autant par la recherche d'objets et de méthodes communes que par le rejet des approches modernes ou positivistes. Ils se forgent peu à peu leurs lieux de diffusion institutionnelle en s'emparant notamment de revues reconnues ou en en créant (Journal of Consumer Research (JCR), European Journal of Marketing, Culture, Markets, and Consumption, International Journal of Research in Marketing, Journal of Consumer Culture, Journal of Marketing, Journal of Material Culture, Research in Consumer Behavior, Journal of Retailing) et en organisant des colloques en Europe, en Asie et en Amérique latine et du Nord par l'entremise de l'Association for Consumer Research (ACR) ou par le groupe CCT qui organise un colloque annuel aux États-Unis depuis 2006.

04. " II est à noter que ce panorama est plus francophone (français mais aussi belge et canadien) que français. » (Özçağlar-Toulouse \& Cova, 2008)

05. Il est intéressant de remarquer que d'après Featherstone (1991) citant Hassan (1985) et Kohler (1977), la première utilisation du terme postmodernisme peut être retracée aux années 1930 dans les travaux de Federico de Onis, écrivain espagnol. II gagne ensuite en popularité auprès de jeunes artistes new-yorkais dans les années 60 
Le courant francophone tel que présenté par Özçağlar-Toulouse \& Cova (2008) s'est davantage développé par intégration aux événements préexistants comme le congrès annuel de l'Association Française de Marketing (AFM). Par la suite, dès 1996, les Journées de Recherche en Marketing de Bourgogne puis, dès 2002, les Journées Normandes de la Consommation deviennent des lieux de rendez-vous pour les chercheurs français en CCT. Une nouvelle revue francophone est d'ailleurs en création actuellement. Enfin, des collaborations transatlantiques permettent d'échanger avec des chercheurs anglophones: Bernard Cova publie et communique auprès de la communauté anglophone, Éric Arnould correspond avec Dominique Desjeux. Et bien que l'apport des recherches francophones aux CCT reste somme toute limité, il les enrichit par le développement des concepts autour de la sociosémiotique et de la valeur de lien [social] (Özçağlar-Toulouse \& Cova, 2008).

\section{Axes de recherche}

Sans revenir sur les apports des CCT francophones, pour lesquels la lecture de l'article d'Özçağlar-Toulouse \& Cova (2008) est édifiante, nous nous attarderons maintenant sur les axes programmatiques de recherche qu'identifient Arnould \&Thompson $(2005,2007)$.Ils nous en proposent quatre qui recoupent sous différents angles les questions liées à la consommation en tant que processus socio-culturel avec les projets de constructions identitaires du consommateur (1), les cultures dans les différents marchés (2), les modèles socio-historiques de consommation (3) et la médiatisation idéologique par les marchés en rapport avec les stratégies interprétatives des consommateurs (4). Ces catégories, nous préviennent-ils, ne sont pas hermétiques et cherchent simplement à souligner les différentes problématiques traitées dans les CCT.

L'axe des projets de constructions identitaires des consommateurs met l'accent sur la manière et les objectifs poursuivis par les consommateurs lorsqu'ils se construisent une ou plusieurs identités en fonction des marchés dans lesquels ils s'inscrivent. Le marché est en effet devenu la source principale des ressources symboliques qui permettent aux consommateurs de se raconter à soi et à autrui en se construisant une identité (Belk, 1988; Hill, 1991; Hill \& Stamey, 1990; Holt, 2002; Levy, 1981). Les identités ainsi produites ont une finalité (Mick \& Buhl, 1992; Schau \& Gilly, 2003) qui peut être implicite ou pas (Arnould \& Price, 1993 ; Thompson \& Tambyah, 1999) et marquée par des conflits internes (Hirschman, 1992 ; Mick \& Fournier, 1998 ; Murray, 2002 ; O'Guinn \& Faber, 1989 ; Otnes et al., 1997 ; Thompson, 1996) qui engendrent des stratégies d'adaptation pour réussir à maintenir une représentation intégrée de soi (Schau \& Gilly, 2003). Ces constructions identitaires sont structurées par la relation des consommateurs au marché qui leur propose des rôles à investir (Kozinets, 2001 ; Belk et al., 2003 ; Murray, 2002 ; Thompson \& Tabyah, 1999) au point que, même lorsque les individus optent pour des actes de consommation non-coformistes, authentiques et 
non consuméristes, la symbolique marchande est au cœur de leurs identités (Holt, 2002 ; Grayson \& Martinec, 2004).

L'axe sur les cultures de consommation (Kozinets, 2001) présente les individus non plus comme des vecteurs mais comme des producteurs de culture. L'accent est mis ici sur manière dont l'émergence de pratiques de consommation reconfigure réflexivement les structures culturelles d'action et d'interprétation des individus. Les chercheurs s'interrogent ici sur les développements culturels dans des milieux spécifiques qu'ils s'articulent autour d'une zone géographique ou d'un intérêt de consommation. Dans le premier cas, Arnould \& Thompson identifient des recherches de ce type sur l'Amérique du Nord (McCracken, 1986 ; Witowski, 1989), l'Afrique (Arnould, 1989 ; Bonsu \& Belk, 2003), l'Asie (Appelbaum \& Jordt, 1996 ; Joy, 2001 ; Tse, Belk \& Zhou, 1989) et l'Europe de l'Est (Coulter et al., 2003). Dans le deuxième, on observe comment la dynamique sociale crée des espaces culturels distinctifs bien que parfois transitoires (Arnould \& Price, 1993 ; Belk \& Kosta, 1998 ; Kozinets, 2002) par le partage d'un intérêt pour un objet de consommation (Belk \& Costa, 2008 ; Kozinets, 2002 ; Schouten \& McAlexander, 1995). Ce courant se construit sur la posture postmoderne et l'idée de néotribalisme de Maffesoli (1988, 1996), selon qui les bases traditionnelles de la socialisation ont été sapées au profit d'un individualisme radical orienté vers une quête incessante de différenciation personnelle et d'autonomie qui amène, paradoxalement, les individus à se construire des identités collectives éphémères et à participer à des rituels de solidarité ancrés dans des styles de vie et des loisirs communs (Cova, 1997 ; Firat \& Venkatesh, 1995 ; Muñiz \& O’Guinn, 2001). Cela permet aux individus de se reconnaître grâce à l'étalage et à la recomposition de capitaux culturels localisés (Belf \& Kosta, 1998 ; Celsi et al., 1993; Kates, 2002 ; Kozinets, 2001, 2002 ; McAlexander, Schouten \& Koenig, 2002) qui leur permettent de se définir par le rejet d'une culture dominante (Brown, Kozinets \& Sherry, 2003 ; Kates, 2002 ; Muñiz \& O’Guinn, 2001 ; Muñiz \& Schau, 2005 ; Schouten \& McAlexander, 1995).

La modélisation socio-historique de la consommation, troisième axe programmatique proposé par Arnould \& Thompson, se penche sur des éléments de structures sociales, telles que la classe (Allen, 2002 ; Holt, 1997, 1998 ; Wallendorf, 2001), l'ethnie (Belk, 1992 ; Mehta \& Belk, 1991 ; Reilly \& Wallendorf, 1987 ; Wallendorf \& Reilly 1983), le sexe (Bristor \& Fischer, 1993 ; Dobscha \& Ozanne, 2001 ; Fischer \& Arnold, 1990 ; Thompson, 1996 ; Thompson \& Haytko, 1997 ; Thompson, Locander \& Pollio, 1990) et d'autres encore, qui influencent la consommation. Les chercheurs s'interrogent alors sur ce qu'est la société de consommation et comment elle se construit et se développe. Ils visent ici à mettre en évidence la relation entre les structures sociales et les expériences, les systèmes de croyances et les pratiques des consommateurs. 
Enfin, les travaux sur les idéologies de consommation et les stratégies interprétatives $d u$ consommateur portent une attention particulière aux systèmes de signification que véhiculent les consommateurs. L'approche est ici délibérément critique et orientée vers les médias. Elle s'éloigne en fait de la recherche sur les consommateurs (Fiske, 1989 ; Hall, 1993 ; Lears, 1994 ; Twitchelle, 1996 ; Dawson, 2003). Les chercheurs s'intéressent à la normativité des messages transmis par les médias au sujet de la consommation (Hirschman, 1988), à la réception et interprétation de ces messages (Hetrick \& Lozada, 1994 ; Hirschman \& Thompson, 1997 ; Murray \& Ozanne, 1991 ; Murray, Ozanne \& Shapiro, 1994). Les consommateurs sont donc ici des agents interprétatifs qui adhérent complètement aux modèles dominants proposés par les médias ou qui les rejettent volontairement. Ce dernier type d'agent donne lieu non seulement à diverses formes identitaires, mais s'inscrivent dans une critique des institutions capitalistes et marketing (Holt, 2002 ; Kozinets, 2002 ; Kozinets \& Haldeman, 2004 ; Murray, 2002 ; Thompson, 2004). Ces recherches se penchent aussi bien sur l'influence de la mondialisation sur les systèmes de production de cultures, que sur celle des industries. La question est de savoir comment les structures orientent les modes de consommation. Les approches sémiotiques et littéraires y sont favorisées afin d'établir une analyse de la symbolique et des idéologies mises en œuvre dans ces structures (Escalas \& Stern, 2003 ; Hirschman, 1988, 1990 ; Holbrook \& Grayson, 1986 ; McQuarrie and Mick, 1996 ; Mick, 1986 ; Sherry \& Camargo, 1987 ; Stern 1993, 1995, 1996 ; Scott. 1990, 1994a, 1994b).

\section{Cheminements épistémologiques}

Une des différences importantes entre le stade de développement des Sciences de gestion, dont les recherches en marketing, et celui des SIC, selon Courbet (2001), est que le premier est à une étape paradigmatique alors que le deuxième en est plutôt à une phase pré-paradigmatique. Nous constatons pourtant que, si les CCT forment partie des recherches en marketing, comme elles remettent fortement en cause les fondements épistémologiques des recherches, elles seraient alors également à inscrire dans une période pré-paradigmatique. Les CCT s'écartent des bases de l'épistémologie positiviste sur lesquelles se sont construites les SG. En reprenant les critères de scientificité que Courbet (2001) a proposés pour comparer SG et SIC, nous allons voir des points de convergences possibles.

- Les composantes syntaxico-sémantiques : le mode de représentation de la réalité.

Les CCT ne cherchent plus directement à identifier un système de causalités exploitable managérialement, mais plutôt à explorer « la distribution hétérogène du sens et la multiplicité des regroupements culturels qui existent dans le cadre socio-historique plus large de la mondialisation et du capitalisme de marché ${ }^{6}$ 06. Notre traduction. 
(Arnould \& Thompson, 2005, p. 869). Les approches qualitatives semblent alors plus à même de rendre compte de cette complexité faisant appel chez les chercheurs à des capacités d'interprétation fines dans des contextes microsociaux. À cet effet, le langage mathématique des SG cède la place au langage naturel des Sciences humaines et sociales dont font partie les SIC. ${ }^{7}$

- Les composantes empiriques : le lien connaissances-réalité

En raison de l'accent mis sur les dimensions culturelles de la consommation dans des contextes micro-sociaux, ou perspectives émiques, et la prédominance des approches interprétatives, les chercheurs favorisent alors des démarches inductives. Celles-ci ne sont d'ailleurs pas sans leur poser de difficultés par rapport à la doxa dominante en SG lorsqu'il s'agit de redécouvrir des mécanismes du constructivisme scientifique : "Ici, Lincoln E Guba (2003) nous éclairent sur les critères pour juger de la "réalité" et la validité [de conclusions de recherche], et se tournent vers le consensus dans la communauté pour valider ce qui est réel, ce qui est utile et ce qui a un sens ${ }^{8}$ (Moisander, Peñalosa \& Valtonen, 2009).

- Critères de validations pragmatiques

L'utilité socio-économique, ou pertinence managériale, n'est plus l'objectif principal des CCT. En tout cas, elle ne l'est pas telle qu'elle a été définie dans le cadre du paradigme du choix rationnel (Arnould \& Thompson, 2005). Les visées des recherches en CCT étant compréhensives, les implications opérationnelles sont alors plus difficiles à établir. Et cela sera le cas tant que l'utilité en SG se mesurera par les seules conséquences économiques des résultats de recherche. Finalement, les CCT réinscrivent le marketing dans une science de la société 9 .

\section{Conclusion}

Les CCT, bien qu'aujourd'hui davantage ancrées dans les SG, offrent des ouvertures thématiques et institutionnelles intéressantes pour les SIC. Ces dernières, sans abandonner les postures critiques qu'elles héritent en partie de l'École de Francfort, peuvent remettre dans leurs programmes de recherche les questions liées à la consommation tout en conservant des objets, des approches, des méthodes et des finalités qui lui restent habituelles. En effet, les axes programmatiques proposés par Arnould \& Thompson $(2005,2007)$ auraient très bien pu s'inscrire dans notre discipline. Les projets identitaires, les cultures et les idéologies de la consommation, les stratégies interprétatives des acteurs sont déjà des objets de recherche familiers en SIC. De plus, et bien

7. Bien qu'Arnould \& Thompson (2005) refusent de distinguer les CCT des SG par des épithètes tels que post-positiviste et interprétatif et prônent une adaptation méthodologique (quantitative ou qualitative), d'autres chercheurs continuent de militer pour une distinction entre les postures positivistes et interprétatives (Moisander, Peñalosa \& Valtonen, 2009).

08. Notre traduction.

09. Sans pour autant être a priori une sociologie de la consommation, mais cela devra être l'objet d'une autre discussion. 
que nous ne l'ayons pas évoqué ici, les différentes déclinaisons des médias, des plus classiques (presse, télévision) aux plus récentes (internet et les réseaux sociaux numériques), occupent une place centrale dans ces nouvelles approches des consommateurs.

La communauté des SIC peut s'intéresser aux consommateurs comme en témoignent le numéro 56 de Sciences de la Société (2002) ou encore le numéro 27 de Communication E Organisation (2005) qui ont pu servir en France de point de jonction avec la communauté des SG. Mais ces efforts semblent ponctuels. Revivrons-nous une découverte et un engouement tardif comme pour les Cultural Studies (cf. Mattelart et Neveu, 2003) ? Les SG s'ouvrent à d'autres disciplines et évoluent dans leurs épistémologies vers des positions qui facilitent les possibilités de dialogue. Leur laisser ou les abandonner à ces questions serait alors une aberration stratégique au risque de nous fermer à une partie des phénomènes de communication que nous étudions, ceux de la société de consommation.

\section{BIBLIOGRAPHI}

ARNOULD E. J., « Consumer culture theory: retrospect and prospect », in Ekström K M, Brenbeck H (Eds), European Advances in Consumer Research, ACR, Duluth, MN, 7, 2006, pp. 605-7.

ARNOULD E. J., THOMPSON C J, « Consumer Culture Theory (CCT): Twenty Years of Research », Journal of Consumer Research, 31, 2005 (March), p. 868-882.

ARNOULD E. J., THOMPSON C. J., « Consumer Culture Theory (and we really mean theoretics) : dilemmas and opportunities posed by an academic branding strategy » In Belk R W, Sherry Jr J F (Ed), Consumer Culture Theory, Research in consumer behaviour, volume XI, 2007, p. 3-22.

BELK R. W., COSTA J. A., «The Mountain Myth: A Contemporary Consuming Fantasy ", Journal of Consumer Research, 25, 1998 (December), pp. 218-240.

BELK R. W, GER G., ASKEGAARD S., «The Fire of Desire: A Multisited Inquiry into Consumer Passion ", Journal of Consumer Research, 30, 2003 (December), pp. 326352.

BERNARD F., JOULE R.-V., «Le pluralisme méthodologique en sciences de l'information et de la communication à l'épreuve de la communication engageante ", Questions de communication, (7), 2005, p. 85-207.

BONSU S. K., BELK R. W., « Do Not Go Cheaply into That Good Night: Death Ritual Consumption in Asante Ghana ", Journal of Consumer Research, 30, 2003 (June), pp. 41-55.

BROWN S., « Postmodern Marketing? », European Journal of Marketing, 27 (4), 1993, pp.19-34.

CELSI R., ROSE R., LEIGH T., "An Exploration of High-Risk Leisure Consumption through Skydiving ", Journal of Consumer Research, 20, 1993 (June), pp. 1-21. 
COURBET D., «Comparaison épistémologique des recherches en SIC et sciences de gestion dans le domaine de la communication externe, divergences et terrain commun ", in Actes du colloque La communication d'entreprise: regards croisés sciences de gestion et sciences de l'information et de la communication, Nice, 6-7 décembre 2001.

CUCHE D., La notion de culture dans les sciences sociales, Paris, La Découverte, 2004.

DACHEUX N., "Étudier le marketing à la lumière de la communication », L'Année sociologique, 2001/2, Vol.51, p. 411-427.

DAWSON M., The Consumer Trap: Big Business Marketing in American Life, Champaign, University of Illinois Press, 2003.

DOBSCHA S., OZANNE J. L., "An Ecofeminist Analysis of Environmentally Sensitive Women Using Qualitative Methodology: The Emancipatory Potential of an Ecological Life ", Journal of Public Policy and Marketing, 20, 2001 (Fall), pp. 201-214.

FIRAT A F., VENKATESH A, «Liberatory Postmodernism and the Reenchantment of Consumption ", Journal of Consumer Research, 22, 1995 (December), pp. 239-67.

FISCHER E, ARNOLD S. J., " More than a Labor of Love: Gender Roles and Christmas Gift Shopping », Journal of Consumer Research, 17, 1990 (December), pp. 333345.

FLORIS B., «L'ingénierie symbolique dans les relations professionnelles. De la lutte des classes au management participatif. ", in Le Moënne Ch (coord.), Communications d'entreprises et d'organisations, Rennes, PUR, 1998, pp. 79-93.

FLORIS B., "Communication et gestion symbolique dans le marketing ", Les Enjeux de l'information et de la communication, (2), 2001, [en ligne]

http://www.ugrenoble3.fr/les_enjeux/2001/Floris/home.html, page consultée le 23/02/2010.

GRAYSON K., MARTINEC R., « Consumer Perceptions of Iconicity and Indexicality and Their Influence on Assessments of Authentic Market Offerings ", Journal of Consumer Research, 31, 2004 (September), p. 296-313.

HALL S., " Encoding, Decoding " in The Cultural Studies Reader, New York: Routledge, ed. Simon During, 1993, pp. 90-103.

HILL R.P., STAMEY M., "The Homeless in America: An Examination of Possessions and Consumption Behaviors ", Journal of Consumer Research, 17, 1990 (December), pp. 303-321.

HILL R.P., « HomelessWomen, Special Possessions, and the Meaning of Home: An Ethnographic Case Study ", Journal of Consumer Research, 18, 1991 (December), pp. 298310.

HIRSCHMAN E. C., THOMPSON C. J., «Why Media Matter: Towards a Richer Understanding of Consumers' Relationships with Advertising and Mass Media ", Journal of Advertising, 26, 1997 (Spring), pp. 43-60.

HIRSCHMAN E.C., "The Ideology of Consumption: A Structural-Syntactical Analysis of 'Dallas' and 'Dynasty' ", Journal of Consumer Research, 15, 1988 (December), pp. 344-359.

HIRSCHMAN E.C., "Secular Immortality and the American Ideology of Affluence ", Journal of Consumer Research, 17, 1990 (June), pp. 31-42. 
HOLBROOK M. B., GRAYSON M. W., "The Semiology of Cinematic Consumption: Symbolic Consumer Behavior in Out of Africa ", Journal of Consumer Research, 13, 1986 (December), pp. 374-381.

HOLT D. B., « Poststructuralist Lifestyle Analysis: Conceptualizing the Social Patterning of Consumption », Journal of Consumer Research, 23, 1997 (March), pp. 326-350.

HOLT D. B., « Does Cultural Capital Structure American Consumption? », Journal of Consumer Research, 25, 1998 (June), pp. 1-26.

HOLT D. B., «Why Do Brands Cause Trouble? A Dialectical Theory of Consumer Culture and Branding », Journal of Consumer Research, 29, 2002 (June), pp. 70-90.

JOY A S, " Gift Giving in Hong Kong and the Continuum of Social Ties ", Journal of Consumer Research, 28, 2001 (September), pp. 239-256.

KATES S. M., «The Protean Quality of Subcultural Consumption: An Ethnographic Account of Gay Consumers ", Journal of Consumer Research, 29, 2002 (December), pp. 383-399.

KOZINETS R. V., «Utopian Enterprise: Articulating the Meaning of Star Trek's Culture of Consumption », Journal of Consumer Research, 28, 2001 (June), pp. 67-89.

KOZINETS R. V., «Can Consumers Escape the Market? Emancipatory Illuminations from Burning Man ", Journal of Consumer Research, 29, 2002 (June), pp. 20-38.

KOZINETS R. V., HANDELMAN J. M., "Adversaries of Consumption: Consumer Movements, Activism, and Ideology ", Journal of Consumer Research, 31, 2004 (December), pp. 691-704.

KROEBER A. L., KLUCKHOHN C., Culture: A Critical Review of Concepts and Definitions. New York, Vintage Books, 1952.

LEARS J., Fables of Abundance, New York, Basic Books, 1994.

LEVY S.J., «Interpreting Consumer Mythology: A Structural Approach to Consumer Behavior », Journal of Marketing, 45, 1981 (Summer), pp. 49-61.

LINCOLN Y. S., GUBA E G., « Paradigmatic controversies, contradictions and emerging confluences », in Dentzin N K, Lincoln Y S (eds.), The Landscape of Qualitative Research, London, Sage, 253-91.

MATTELART A., NEVEU É., Introduction aux Cultural Studies, Paris, La Découverte, 2003.

MCALEXANDER J. H., SCHOUTEN J. W., KOENIG H, « Building Brand Community » Journal of Marketing, 66, 2002 (January), pp. 38-54.

MCQUARRIE E. F., MICK D. G., « Figures of Rhetoric in Advertising Language », Journal of Consumer Research, 22, 1996 (March), pp. 424-436.

MEHTA R., BELK R. W., " Artifacts, Identity, and Transition: Favorite Possessions of Indians and Indian Immigrants to the United States ", Journal of Consumer Research, 17, 1991 (March), pp. 398-411.

MICK D. G., " Consumer Research and Semiotics: Exploring the Morphology of Signs, Symbols, and Significance ", Journal of Consumer Research, 13, 1986 (September), pp. 196-213. 
MICK D. G., FOURNIER S, « Paradoxes of Technology: Consumer Cognizance, Emotions, and Coping Strategies " Journal of Consumer Research, 25, 1998 (September), pp. 123-143.

MOISANDER J., PEÑALOZA ., VALTONEN, «From CCT to CCC: Building consumer culture community ", in Sherry Jr J F, Fischer E (Ed), Explorations in consumer culture theory, Routledge, Oxon, 2009, pp. 7-33.

MUÑIZ A., O'GUINN T. C., « Brand Community ", Journal of Consumer Research, 27, 2001 (March), pp. 412-432.

MUÑIZ A., SCHAU H., "Religiosity in the Abandoned Apple Newton Brand Community ", Journal of Consumer Research, 31, 2005 (March), pp. 737-747.

MURRAY J. B., "The Politics of Consumption: A Re-Inquiry on Thompson and Haytko's (1997) 'Speaking of Fashion' », Journal of Consumer Research, 29, 2002 (December), pp. 427-440.

MURRAY J. B., OZANNE J. L., "The Critical Imagination: Emancipatory Interests in Consumer Research ", Journal of Consumer Research, 18, 1991 (September), pp. 129144.

MURRAY J., OZANNE J., L., SHAPIRO J., « Revitalizing the Critical Imagination: Unleashing the Crouched Tiger», Journal of Consumer Research, 21, 1994 (December), pp. 559-565.

O'GUINN T. C., FABER R. J., " Compulsive Buying: A Phenomenological Exploration ", Journal of Consumer Research, 16, 1989 (September), pp. 147-157.

OTNES C., LOWREY T., SHRUM L. J., « Toward an Understanding of Consumer Ambivalence ", Journal of Consumer Research, 241997 (June), pp. 80-93.

ÖZÇAGLAR-TOULOUSE N., COVA B., «Contributions françaises à la CCT: histoire et concepts clés ", 13 Journées de Recherche en Marketing de Bourgogne, Dijon, 13 et 14 novembre 2008, [en ligne] http://www.u-bourgogne.fr/LEG/CERMAB/zoutils/ documents/actesJRMB/JRMB13-2008/Ozcaglar\%20Cova.pdf, consulté le 15/04/2010.

REILLY M. D., WALLENDORF M., "A Comparison of Group Differences in Food Consumption Using Household Refuse," Journal of Consumer Research, 14, 1987 (September), pp. 289-294.

SCHAU H. J., GILLY M. C., «We Are What We Post? Self-Presentation in Personal Web Space », Journal of Consumer Research, 30, 2003 (December), pp. 385-404.

SCHOUTEN J., MCALEXANDER J. H., "Subcultures of Consumption: An Ethnography of the New Bikers ", Journal of Consumer Research, 22, 1995 (June), pp. 4361.

SCOTT L. M., « Understanding Jingles and Needledrop: A Rhetorical Approach to Music in Advertising ", Journal of Consumer Research, 17, 1990 (September), pp. 223-236.

SCOTT L. M., «The Bridge from Text to Mind: Adapting Reader-Response Theory to Consumer Research ", Journal of Consumer Research, 21, 1994a (December), pp. 461480.

SCOTT L. M., «Images in Advertising: The Need for a Theory of Visual Rhetoric », Journal of Consumer Research, 21, 1994b (September), pp. 252-273. 


\section{$\mathrm{C} \& \mathrm{O} \mathrm{n}^{\circ} 37$}

SHERRY J. F., CAMARGO E., «'May Your Life Be Marvelous': English Language Labeling and the Semiotics of Japanese Promotion ", Journal of Consumer Research, 14, 1987 (September), pp. 174-188.

STERN B. B., «Feminist Literary Criticism and the Deconstruction of Ads: Overview and Illustrative Analysis », Journal of Consumer Research, 19, 1993 (March), pp. 556-566.

STERN B. B., «Consumer Myths: Frye's Taxonomy and the Structural Analysis of Consumption Text », Journal of Consumer Research, 22, 1995 (September), pp. 165-185.

STERN B. B., " Deconstructive Strategy and Consumer Research: Concepts and Illustrative Exemplar », Journal of Consumer Research, 23, 1996 (September), pp. 136-147.

THOMPSON C. J., " Caring Consumers: Gendered Consumption Meanings and the Juggling Lifestyle ", Journal of Consumer Research, 22, 1996 (March), pp. 388-407.

THOMPSON C. J., HAYTKO D. L., "Speaking of Fashion: Consumers' Uses of Fashion Discourses and the Appropriation of Countervailing Cultural Meanings ", Journal of Consumer Research, 24, 1997 (June), pp. 15-42.

THOMPSON C. J., TAMBYAH S. K., « Trying to Be Cosmopolitan », Journal of Consumer Research, 26, 1999 (December), pp. 214-241.

THOMPSON C. J., LOCANDER W. B., POLLIO H R, « The Lived Meaning of Free Choice: An Existential-Phenomenological Description of Everyday Consumer Experiences of Contemporary Married Women ", Journal of Consumer Research, 17, 1990 (December), pp. 346-361.

TSE D. K., BELK R.W, ZHOU N., «Becoming a Consumer Society: A Longitudinal and Cross-Cultural Content of Analysis of Print Ads from Hong Kong, the People's Republic of China, and Taiwan ", Journal of Consumer Research, 15, 1989 (September), pp. $457-472$.

TWITCHELL J., Adcult USA: The Triumph of Advertising in American Culture, New York, Columbia University Press, 1996.

WALLENDORF M., « Literally Literacy », Journal of Consumer Research, 27, 2001 (March), pp. 505-511.

WALLENDORF M., REILLY M. D., " Ethnic Migration, Assimilation, and Consumption ", Journal of Consumer Research, 10, 1983 (December), pp. 292-302.

WITKOWSKI T. H., " Colonial Consumers in Revolt: Buyer Values in Behavior during the Nonimportation Movement, 1764-1776 ", Journal of Consumer Research 16, 1989 (September), pp. 216-226.

Revue Sciences de la Société, «Les figures sociales du client », Cochoy F (coord.), 56, Toulouse, PUM, mai 2002.

Revue Communication E Organisation, "Fidélisation et personnalisation. Les nouvelles formes de relations consommateurs/entreprises", Laborde A (coord), Bordeaux, GREC/O, 27, juin 2005.

Résumé : La recherche en marketing a pris un tournant interprétatif et critique sur ces vingt-cinq dernières années en étudiant notamment les dimensions culturelles des phénomènes de consommation à un niveau davantage micro-sociologique. Son 
cheminement épistémologique et méthodologique ainsi que le programme de recherche de ce courant, qui peut être identifié sous l'appellation récente de Consumer Culture Theory, peut être une invitation aux Sciences de l'information et de la communication pour réinvestir, sans s'enfermer dans une posture exclusivement critique, l'étude des consommateurs.

Mots-clés : consommateur, Consumer Culture Theory, critique, épistémologie, interprétatif, marketing.

\begin{abstract}
On this last twenty five years, marketing research has evolved towards interpretive and critical perspectives by studying consumption cultural dimensions on a more micro-sociological level. The epistemological and methodological journey as well as the research programm of what may now be called Consumer Culture Theory shall be inviting for the Information and communication sciences to study, and not only from a critical perspective, market and consumer phenomena.
\end{abstract}

Keywords : consumer, Consumer Culture Theory, critical, epistemology, interpretive, marketing 